This item was submitted to Loughborough's Institutional Repository (https://dspace.lboro.ac.uk/) by the author and is made available under the following Creative Commons Licence conditions.

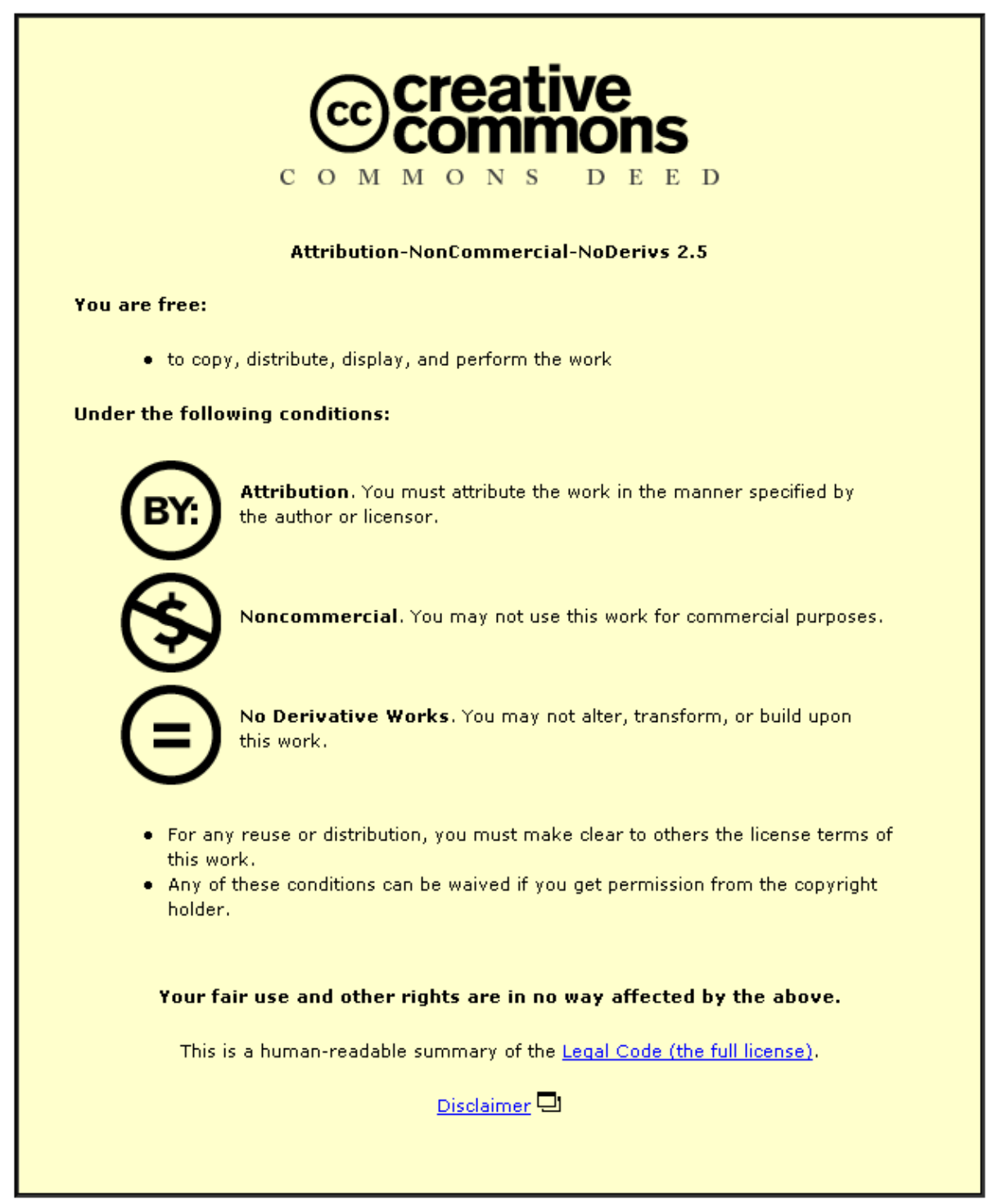

For the full text of this licence, please go to: http://creativecommons.org/licenses/by-nc-nd/2.5/ 


\section{A BLIND LAG-HOPPING ADAPTIVE CHANNEL SHORTENING ALGORITHM BASED UPON SQUARED AUTO-CORRELATION MINIMIZATION (LHSAM)}

\author{
M. Grira \\ Center of Digital Signal Processing \\ Cardiff University, Queen's Buildings \\ PO Box 925, Cardiff CF24 0YF \\ Wales, UK \\ \{griram@cf.ac.uk\}
}

J. A. Chambers

\author{
Advanced Signal Processing Group \\ Dept. of Electronic and Electrical Engineering \\ Loughborough University \\ Leicestershire LE11 3TU, UK \\ \{J.A.Chambers@lboro.ac.uk\}
}

\begin{abstract}
Recent analytical results due to Walsh, Martin and Johnson showed that optimizing the single lag autocorrelation minimization (SLAM) cost does not guarantee convergence to high signal to interference ratio (SIR), an important metric in channel shortening applications. We submit that we can overcome this potential limitation of the SLAM algorithm and retain its computational complexity advantage by minimizing the square of single autocorrelation value with randomly selected lag. Our proposed lag-hopping adaptive channel shortening algorithm based upon squared autocorrelation minimization (LHSAM) has, therefore, low complexity as in the SLAM algorithm and, more importantly, a low average LHSAM cost can guarantee to give a high SIR as for the SAM algorithm. Simulation studies are included to confirm the performance of the LHSAM algorithm.
\end{abstract}

Index Terms - Adaptive filtering, channel shortening, multicarrier modulation

\section{INTRODUCTION}

In multicarrier modulation (MCM) systems, such as asymmetrical digital subscriber line (ADSL) transceivers, each symbol consists of samples to be transmitted to the receiver plus a cyclic prefix (CP) of length $\mathrm{v}$ [1]. The CP is the last v samples of the original $\mathrm{N}$ samples to be transmitted. The $\mathrm{CP}$ is inserted between blocks to combat inter-symbol interference (ISI) and inter-channel interference (ICI). The length of the $\mathrm{CP}$ should at least be equal to the order of the channel impulse response. At the receiver the $\mathrm{CP}$ is removed, and the remaining $\mathrm{N}$ samples are then processed by the receiver. Since the efficiency of the transceiver is reduced by the introduction of the $\mathrm{CP}$ it is therefore desirable either to make $\mathrm{v}$ as small as possible or to choose a large $\mathrm{N}$. Selecting large $\mathrm{N}$ will increase the computational complexity, system delay, and memory requirements of the transceiver. To overcome these problems a short time-domain equalizer (TEQ), usually an FIR filter, can be placed in the front end of the receiver, to shorten the impulse response of the effective channel. The length of the shortened impulse response filter and $\mathrm{CP}$ are usually fixed a priori and not changed from channel to channel. A low complexity blind adaptive algorithm to design a TEQ, called sumsquared auto-correlation minimization (SAM) was proposed in [2] which achieves channel shortening by minimizing the sum-squared autocorrelation terms of the effective channel impulse response outside a window of a desired length. The drawback with SAM is that it has a significant computational complexity. SLAM [3], on the other hand, achieves channel shortening by minimizing the squared value of only a single autocorrelation at a lag greater than the guard interval. The drawback with SLAM is that even guaranteeing convergence of the SLAM cost to low values does not necessarily guarantee convergence to high SIRs [4]. Our contribution is therefore to propose a new channel shortening algorithm with random lag selection which retains the complexity advantage of SLAM whilst mitigating its SIR problem.

The paper is organized as follows. Section 2 gives the system model. Section 3 discusses the idea of minimizing the autocorrelation at a random lag. Section 4 develops the gradientdescent implementation of the LHSAM algorithm. Section 5 discusses the SIR performance for the SAM, SLAM, and LHSAM algorithms. Section 6 provides the comparative simulations between SAM and LHSAM and in Section 7 conclusions are drawn.

\section{SYSTEM MODEL}

The system model is shown in Figure (1). The input signal $x(n)$ is the source sequence to be transmitted through a linear finite-impulse-response (FIR) channel $\mathbf{h}$ of length $\left(L_{h}+\right.$ 1)taps, $r(n)$ is the received signal, which will be filtered through an $\left(L_{w}+1\right)$-tap TEQ with an impulse response vector $\mathbf{w}$ to obtain the output sequence $y(n)$. For convenience in this work we assume real signals but generalization to the complex case is straight-forward. We denote $\mathbf{c}=\mathbf{h} * \mathbf{w}$ as the shortened or 


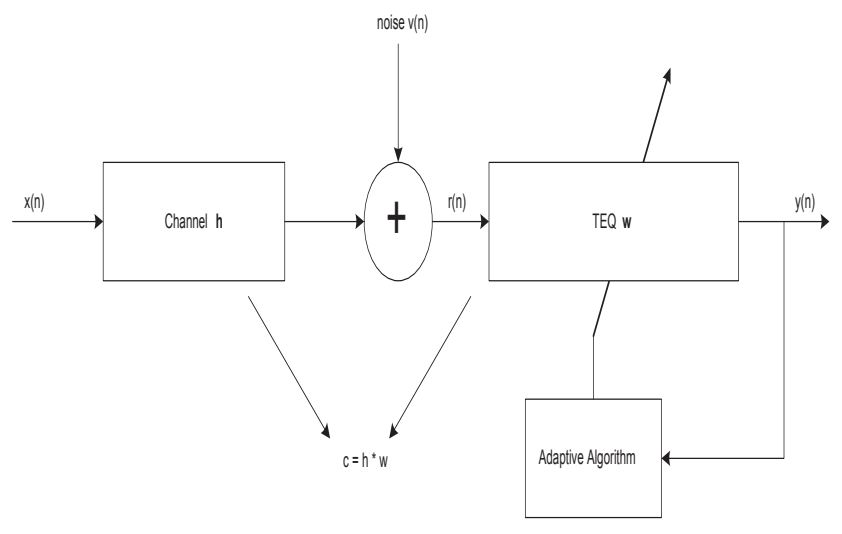

System model for blind adaptive channel shortening

Fig. 1. System model for blind adaptive channel shortening.

effective channel assuming $\mathbf{w}$ is in steady-state where $*$ denotes discrete time convolution. We also assume that $2 L_{c}<$ $N_{f f t}$ holds, where $L_{c}$ is the order of effective channel and $N_{f f t}$ is the FFT size [2]. The signal $v(n)$ is a zero-mean, i.i.d., noise sequence, uncorrelated with the source sequence with variance $\sigma_{v}^{2}$. The received sequence $r(n)$ is

$$
r(n)=\sum_{k=0}^{L_{h}} h(k) x(n-k)+v(n)
$$

and the output of the TEQ $y(n)$ is given by

$$
y(n)=\sum_{k=0}^{L_{w}} w(k) r(n-k)=\mathbf{w}^{T} \mathbf{r}_{n}
$$

where $\mathbf{r}_{n}=\left[\begin{array}{ll}r(n) & r(n-1) \cdots r\left(n-L_{w}\right)\end{array}\right]^{T}$ and $\mathbf{w}$ is the impulse response vector of the TEQ $\mathbf{w}=\left[\begin{array}{lll}w_{0} & w_{1} & w_{2} \cdots w_{L_{w}}\end{array}\right]^{T}$.

\section{SAM AND SLAM COST FUNCTIONS}

The idea of SAM is based on the fact that for the effective channel $\mathbf{c}$ to have zero taps outside a window of size $(v+1)$, its autocorrelation values should be zero outside a window of size $(2 v+1)$. In SAM the auto-correlation sequence of the combined channel-equalizer impulse response is given by

$$
R_{c c}(l)=\sum_{k=0}^{L_{c}} c(k) c(k-l)
$$

and for a shortened channel, it must satisfy

$$
R_{c c}(l)=0, \forall|l|>v
$$

Then the cost function $J_{v+1}$ in SAM is defined based upon minimizing the sum-squared auto-correlation terms, i.e.,

$$
J_{v+1}=\sum_{l=v+1}^{L_{c}} R_{c c}(l)^{2}
$$

On the other hand, SLAM is based on the fact that a single autocorrelation at a lag greater than the guard interval is a measure of the presence of the channel outside the desired guard interval, therefore minimizing only this single autocorrelation also gives the channel shortening effect. This is particularly applicable to subscriber line channels which are essentially minimum phase. In SLAM the auto-correlation sequence of the combined channel-equalizer impulse response is given by

$$
R_{c c}(l)=\sum_{k=0}^{L_{c}} c(k) c(k-l)
$$

and for a shortened channel, it must satisfy

$$
R_{c c}(l)=0, l=v+1
$$

Then the cost function $J_{v+1}$ in SLAM is defined based upon minimizing the squared auto-correlation of the effective channel at lag $l=v+1$, i.e.,

$$
J_{v+1}=R_{c c}(l)^{2}, l=v+1
$$

Recently, however, it has been highlighted in [4] that minimizing (8) does not guarantee high SIR for certain combined channel and shortener responses. To mitigate this problem our contribution is to modify SLAM so that the lag parameter in (8) is chosen at random to lie within the range $v+1, \ldots, L_{c}$, with equal probability of selecting anyone lag, so that on average the cost is identical to (5) when implemented in an adaptive learning algorithm. The computational advantages of SLAM would thereby be retained.

\section{LHSAM ADAPTIVE ALGORITHM}

The steepest gradient-descent algorithm to minimize the SLAM $\operatorname{cost} J_{v+1}$ is

$$
\mathbf{w}^{\text {new }}=\mathbf{w}^{\text {old }}-\mu \nabla_{\mathbf{w}}(E[y(n) y(n-l)])^{2}
$$

where $l$ is a single lag, $\mu$ denotes the step size, and $\nabla_{\mathbf{w}}$ is the gradient with respect to $\mathbf{w}$. We define the instantaneous cost function, where we replace the expectation operation by a moving average over a user-defined window of length $N_{a v g}$

$$
J_{v+1}^{i n s t}(k)=\left\{\sum_{n=k N_{a v g}}^{(k+1) N_{a v g}-1} \frac{y(n) y(n-l)}{N_{a v g}}\right\}^{2}
$$

where $N_{\text {avg }}$ is a design parameter and it should be large enough to give a reliable estimate of the expectation, but no larger, 
as the algorithm complexity is proportional to $N_{\text {avg }}$. The gradient-descent algorithm is given by

$$
\begin{aligned}
& \mathbf{w}(k+1)=\mathbf{w}(k)-2 \mu\left\{\sum_{n=k N_{a v g}}^{(k+1) N_{a v g}-1} \frac{y(n) y(n-l)}{N_{a v g}}\right\} \\
& \quad \times\left\{\nabla_{\mathbf{w}}\left(\sum_{n=k N_{a v g}}^{(k+1) N_{a v g}-1} \frac{y(n) y(n-l)}{N_{a v g}}\right)\right\}
\end{aligned}
$$

which can be simplified to

$$
\begin{array}{r}
\mathbf{w}(k+1)=\mathbf{w}(k)-2 \mu\left\{\sum_{n=k N_{a v g}}^{{ }^{(k+1) N_{a v g}-1}} \frac{y(n) y(n-l)}{N_{a v g}}\right\} \\
\times\left\{\sum_{n=k N_{a v g}}^{(k+1) N_{a v g}-1}\left(\frac{y(n) \mathbf{r}_{n-l}+y(n-l) \mathbf{r}(n)}{N a v g}\right)\right\}
\end{array}
$$

The key defining feature of the LHSAM algorithm is that at each iteration $k$, the lag ' $l$ ' is chosen with equal probability to take on one of the values in the range of $v+1, \ldots, L_{c}$. Most importantly, through learning, the algorithm is in effect operating on a cost function of the form of (5).

\section{SIR PERFORMANCE}

In [4], the authors examine how the signal to interference (SIR) power ratio in the output of the shortener changes when the shortening metrics of SAM, sum absolute autocorrelation minimization (SAAM), and SLAM are used. The SIR is defined to be

$$
S I R:=\frac{\sum_{l=-v}^{v}\left|R_{c c}(l)\right|^{2}}{\sum_{l=-N}^{-v+1}\left|R_{c c}(l)\right|^{2}+\sum_{l=v+1}^{N}\left|R_{c c}(l)\right|^{2}}
$$

Note that the denominator in this expression is the SAM cost, considering those combined z-domain responses $c(z)$ only which satisfy the unit energy constraint, the following relation can be obtained

$$
\begin{aligned}
& S I R(d B)=10 \log _{10}\left(\sum_{l=-v}^{v}\left|R_{c c}(l)\right|^{2}\right)-10 \log _{10}\left(J_{s}\right) \\
& \quad=10 \log _{10}\left(1+2 \sum_{l=1}^{v}\left|R_{c c}(l)\right|^{2}\right)-10 \log _{10}\left(J_{s}\right) \\
& \quad \geq-J_{s}(d B)
\end{aligned}
$$

where $J_{s}$ is the SAM cost function, and a low SAM cost can be guaranteed to give a high SIR at the output of the matched filter. SLAM design affords no such underbound on the SIR performance. For further detail consult [4]. Our proposed algorithm (LHSAM) overcomes the problem of SLAM by choosing the lag randomly, so that a low average LHSAM cost, achieved through recursive learning, will be identical to a low SAM cost which guarantees to give a high SIR at the output of the matched filter, as on the average the proposed algorithm uses all the lags as in SAM.

\section{SIMULATIONS}

The Matlab code at [5] was extended to simulate LHSAM. The cyclic prefix was of length 32 , the FFT size $N_{f f t}=512$, the TEQ had 16 taps and the channels were the test ADSL channel CSA loop 1 available at [6]. The noise was set such that $\sigma_{x}^{2}\|c\|^{2} / \sigma_{v}^{2}=40 d B$ where $\|$.$\| denotes the Euclidean$ norm; and 75 OFDM symbols were used. The step size used for SLAM and LHSAM was 600, in order to achieve convergence in approximately 1000 blocks. All algorithms are compared with the maximum shortening SNR solution [1], which was obtained using the code at [5], and the matched filter bound (MFB) on capacity, which assumes no ICI.

In Figures (2), (3) and (4) the shortened channels are compared with the original channels and all algorithms are confirmed to be effective. The support of the shortened channel is restricted to lie within the first 50 taps. In Figures (5), (6) and (7) the achievable bits per second as a function of the averaging block number, are plotted which show the improved convergence property of LHSAM over SLAM, best performance is achieved at approximately 900 rather than 1010 blocks, due to the nature of the underlying cost function as a function of the parameters of the shortener. The bit rate was determined based on

$$
R=\sum_{i=1}^{N_{f f t}} \log _{2}\left(1+S N R_{i} / \Gamma\right)
$$

The bit rate was computed using a 6-dB margin and a 4.2-dB coding gain. For more details, see [6], and for more details on how the achievable bit rate relates to SAM cost and ICI, see [2]

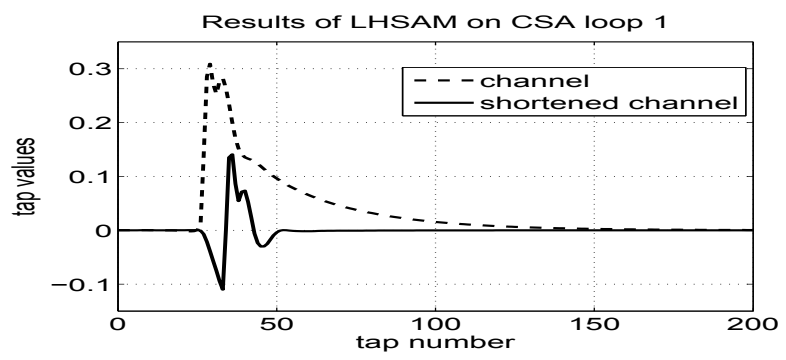

Fig. 2. Channel (dashed) and shortened channel(solid) impulse response of LHSAM algorithm 


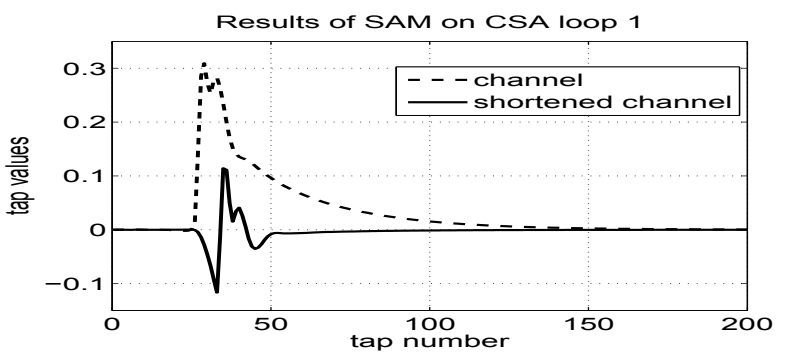

Fig. 3. Channel (dashed) and shortened channel(solid) impulse response of SAM algorithm

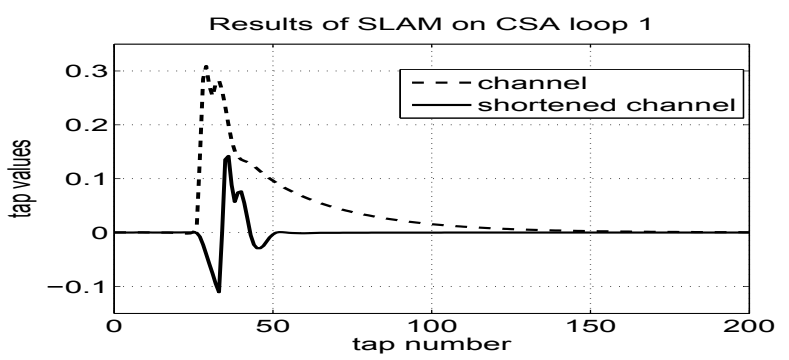

Fig. 4. Channel (dashed) and shortened channel(solid) impulse response of SLAM algorithm

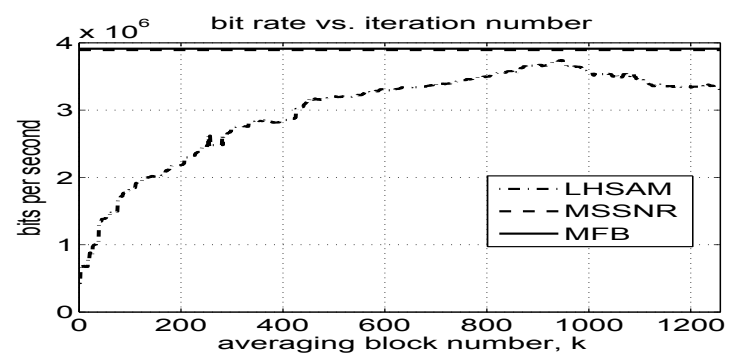

Fig. 5. Achievable bit rate versus iteration number at $40 \mathrm{~dB}$ SNR of LHSAM algorithm

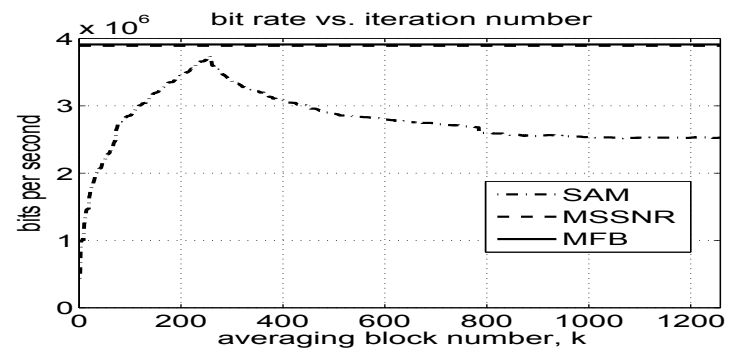

Fig. 6. Achievable bit rate versus iteration number at $40 \mathrm{~dB}$ SNR of SAM algorithm

\section{CONCLUSION}

A new partial update blind channel shortening algorithm has been proposed. The proposed algorithm essentially achieves

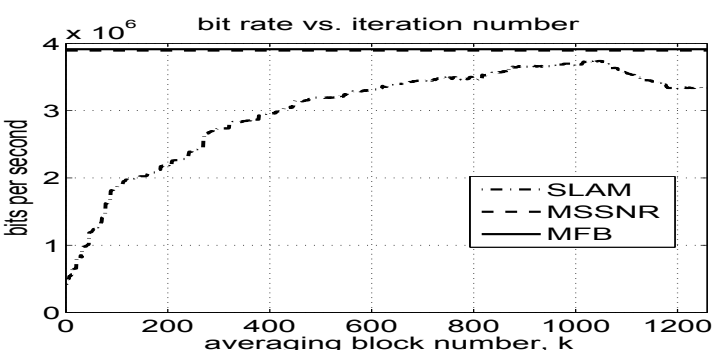

Fig. 7. Achievable bit rate versus iteration number at $40 \mathrm{~dB}$ SNR of SLAM algorithm

the same result in terms of reducing the effective channel length as SLAM. Importantly, however, the disadvantage of SLAM in terms of the SIR performance has been overcome by the proposed algorithm where the proposed algorithm has the advantage of low complexity of SLAM over SAM and also has the advantage of SAM where a low LHSAM cost will be identical to a low SAM cost which guarantees to give a high SIR at the output of the matched filter as on the average the proposed algorithm uses all the lags as in SAM.

\section{REFERENCES}

[1] P. J. W. Melsa, R. C. Younce, and C. E. Rohrs, "Impulse response shortening for discrete multitone transceivers," IEEE Trans. Commun., vol. 44, pp. 1662-1672, December 1996.

[2] J. Balakrishnan, R. K. Martin, and C. R. Johnson, "Blind, adaptive channel shortening by sum-squared auto-correlation minimization (SAM)," IEEE Trans. Signal Processing, vol. 51, pp. 3086-3090, December 2003.

[3] R. Nawaz and J.A. Chambers, "Blind Adaptive Channel Shortening by Single Lag Autocorrelation Minimization (SLAM)," Electronics Letters, vol. 40, pp. 1609-1611, December 2004.

[4] J. M. Walsh, R. K. Martin, and C. R. Johnson, "Convergence and performance issues for autocorrelation based adaptive channel shorteners," Proc. 40th Asilomar Conf. on Signals, Systems, and Computers, 2006.

[5] R. K. Martin, "Matlab code for papers by r. k. martin.[online]. available: http://bard.ece.cornell.edu/matlab/martin/index.html," .

[6] G. Arslan, M. Ding, B. Lu, Z. Shen, and B. L. Evans, "TEQ Design Toolbox. Univ. Texas, Austin, TX. [Online.] Available: http://www.ece.utexas.edu/ bevans/projects/adsl/ dmtteq/dmtteq.html," . 